\title{
Analysis of quality of life in patients with knee osteoarthritis after treatment with autologous leukocyte-rich and leukocyte-poor platelet-rich plasma
}

\author{
Goliuk Ye. ${ }^{1}$, Pshenychnyi T. ${ }^{1}$, Ostapenko T. ${ }^{2}$, Yavorovska V. ${ }^{1}$, Magomedov O. ${ }^{1}$ \\ ${ }^{1}$ State Institute of Traumatology and Orthopedics of the National Academy of Medical Sciences of Ukraine, Kyiv, Ukraine \\ ${ }^{2}$ Ukrainian Scientific Center for Endocrine Surgery, Transplantation of Endocrine Organs and Tissues of Ministry of Health \\ of Ukraine, Kyiv, Ukraine \\ e-mail:viyavr@gmail.com
}

\section{ABSTRACT}

In recent years, there is a growing interest in the application of regenerative medicine methods in the treatment of degenerative injuries of the musculoskeletal system. Knee osteoarthritis (gonarthrosis) is one of the most common reasons of a patient visit to orthopedic surgeons.

THE PURPOSE of the study was to evaluate the quality of life in patients with different stages of gonarthrosis after application of leukocyte-rich platelet-rich plasma (LR-PRP) and leukocyte-poor platelet-rich plasma (LP-PRP).

MATERIALS AND METHODS. The results of the treatment of 48 patients with gonarthrosis grades 1-3 according to Kellgren-Lawrence after intra-articular injection of LR-PRP or LP-PRP were compared. The quality of life questionnaires were used: visual analogue scale, Lysholm Knee Score and Oxford Knee Score before treatment, after the first injection, every 2 weeks before the end of treatment and every 3 months for 1 year.

RESULTS. Evaluation of the treatment results obtained at stages 1 and 2 of knee osteoarthritis showed a positive result for both groups of patients during 12 months of follow-up and at stage 3 a significant improvement was achieved for up to 6 months. There was no significant difference between the results of $L R-P R P$ and $L P-P R P$ injections for 9-12 months. After using LP-PRP treatment, the dynamics of quality of life during the year was stepless and more steady, without periods of regression, while after LR-PRP injections there was a clear significant short-term reduction in the treatment effect for about 6 months, compared to both baseline and values of the previous period at 1 and 2 stages gonarthrosis with further improvement and a stable positive effect for up to 12 months.

CONCLUSIONS. The application of platelet-rich plasma improved the quality of life for 12 months in patients with stage 1-2 gonarthrosis and for 6 months at stage 3. The obtained positive results substantiate the need for further research to determine the prospects for the application of platelet-rich plasma in the complex treatment of knee osteoarthritis compared to other methods.

KEY WORDS: regenerative medicine; platelet-rich plasma; gonarthrosis; quality of life

Regenerative therapy is the newest interdisciplinary field of medicine which studies the use of cell technologies and new materials to ensure reparative or physiological regeneration at various diseases, including musculoskeletal system [34]. Knee osteoarthritis (gonarthrosis) is one of the most common reasons of a patient visit to orthopedic surgeons. Therefore, this pathology is a promising object of application of modern cell technologies. The high efficacy of regenerative medicine and wide range of its application in orthopedics are of interest to clinicians and contribute to its active implementation in routine care. However, the need for fundamental knowledge and understanding of key processes in physiology and regeneration of cartilage, as well as the lack of large clinical trials make regenerative techniques vulnerable and compromise them.

Platelet-rich plasma (PRP) is autologous plasma in which the platelet concentration is increased several times compared to peripheral blood by stepwise centrifugation. Leukocyte-rich platelet-rich plasma (LR-PRP) is autologous plasma with increased compared to baseline concentrations of platelets (> 1 million/ $\mu \mathrm{L}$ ) and leukocytes. Leukocytepoor platelet-rich plasma (LP-PRP) is platelet-rich plasma with a minimal number of white blood cells $[5,6,15]$. 
While platelet concentration is given sufficient attention by clinicians, the role of leukocytes in PRP remains unstudied. It is proved that leukocytes are able to secrete a large number of proinflammatory cytokines, enzymes (interleukin (IL-1 $\beta$ ), metalloproteinase (MMP-9), tumor necrosis factor a (TNF-a), etc. which can cause increased catabolism of the extracellular matrix and inhibition of regeneration processes [16, 25]. In addition, the formation of leukocyte-platelet aggregates (PLAs) plays an important role in the pathogenesis of diseases associated with chronic systemic inflammation. Increased concentrations of PLAs occur in patients with cerebral ischemia [13] at acute coronary syndrome [12, 22, 24], ischemic stroke [13, 24], HIV-1 [26], systemic lupus erythematosus and rheumatoid arthritis [14].

THE PURPOSE of the study was to evaluate the quality of life in patients with knee osteoarthritis (gonarthrosis) at different stages after treatment with leukocyte-rich platelet-rich plasma and leukocyte-poor platelet-rich plasma.

\section{MATERIALS AND METHODS}

The results of the treatment of 48 patients with knee osteoarthritis of different stages ( 25 men and 23 women) treated at the Scientific Center for Tissue and Cell Therapy of the Institute of Traumatology and Orthopedics of the National Academy of Medical Sciences of Ukraine from 2015 to 2020 were evaluated. At the beginning of the treatment the average age of the patients with stage 1 gonarthrosis was $40.5 \pm 7.3$ years, the patients with stage $2-50.6 \pm 9.4$ years, the patients with stage $3-$ $61.9 \pm 7.1$ years. Patients were divided into two groups: the first group included 27 patients treated with LR-PRP, the second -21 patients treated with LP-PRP (Table 1).

Intra-articular injections of 4-5 mL PRP under ultrasound control into the knee joint and 10-12 mL of platelet-poor plasma paraarticularly with an interval of 7 to 14 days between injections were performed. The total treatment course consisted of 5 injections.

All patients underwent a comprehensive clinical and laboratory examination before the treatment. A blood test to assess the white blood cell count and differential as well as erythrocyte sedimentation rate was performed using a hematology analyzer Celltax Alpha (Nihon Kohden, Japan). Biochemical analysis of blood for total protein, bilirubin, urea, creatinine, as well as determination of C-reactive protein, anti-streptoIysin-0, rheumatoid factor was performed using a biochemical analyzer Pentra-400 (HORIBA ABX, France) and the appropriate diagnostic kits of the same manufacturer. Additionally, a rapid immunochromatographic combined tests «PROFITEST» (InTec Products. Inc., China) were performed to detect antibodies to human immunodeficiency virus type 1 and 2 (HIV 1/2), hepatitis B surface antigen ( $\mathrm{HBsAg}$ ), hepatitis B core antibody ( $\mathrm{HBCAb})$, antibodies to hepatitis C (HCV), antibodies to Treponema pallidum.

Absolute contraindications to PRP therapy included: hypofibrinogenemia, fever within a week before the treatment, skin rash in the knees, cancer anamnesis, infectious diseases, anemia with a hemoglobin level less than $100 \mathrm{~g} / \mathrm{L}$, thrombocytopenia less than $1 \cdot 10^{5}$ cells/L, transmissible infections (HIV, hepatitis B, hepatitis C).

LR-PRP and LP-PRP were obtained by differential centrifugation. We separated $50 \mathrm{~mL}$ of venous blood with the dextrose citrate as an anticoagulant into plasma and blood cells at $250 \mathrm{xg}$ for 10 minutes using a centrifuge CM-3 (MICROmed, China). Plasma and pellet containing leukocytes (buffy coat) were collected to obtain LR-PRP. Only plasma was used to obtain LP-PRP. After that, the selected plasma for both products was centrifuged at $2300 \times \mathrm{g}$ for 5 minutes. The platelet pellet was resuspended in 4-5 $\mathrm{mL}$ of platelet-poor plasma, obtained after the second centrifugation.

To evaluate the results of the treatment, we used questionnaires: the visual analog scale (VAS), the Lysholm Knee Score, the Oxford Knee Score. The results were evaluated before the treatment, 2-5 days after
Table 1. Distribution of the patients with gonarthrosis by groups.

\begin{tabular}{ccc|c|c|}
\hline \multirow{2}{*}{$\begin{array}{c}\text { GRADE } \\
\text { (ACCORDING TO J. KELLGREN) }\end{array}$} & TOTAL & \multicolumn{2}{c}{ GROUPS } \\
\cline { 3 - 4 } & & LR-PRP & LP-PRP \\
\hline 1 & 15 & 9 & 6 \\
\hline 2 & 18 & 10 & 8 \\
\hline 3 & 15 & 8 & 7 \\
\hline Total & 48 & 27 & 21 \\
\hline
\end{tabular}

Note: LR-PRP - leukocyte-rich platelet-rich plasma, LP-PRP - leukocyte-poor platelet-rich plasma.

the first injection, then every 2 weeks before the end of the treatment and every 3 months after its completion for at least 12 months from the start of PRP therapy.

The variables were processed by the methods of descriptive statistics and presented as mean and standard deviations. Parametric Fisher's F-test was used as a criterion for reliability after Shapiro-Wilk test for normality. The difference was considered statistically significant at $p \leq 0.05$. Pearson's correlation coefficient was used to determine the linear association between variables.

\section{RESULTS AND DISCUSSION}

\section{The evaluation of the clinical results in patients with stage 1} gonarthrosis.

During the first month, the decrease in pain and increase in the knee functional ability had a positive trend in patients of both groups. In the patients of the first group treated with LR-PRP, the average pain index by the VAS before the treatment was $34.1 \pm 3.8 \mathrm{~mm}$, while in the patients of the second group it was $35.0 \pm 6.3 \mathrm{~mm}$ (Fig. 1). One month later, the pain intensity decreased to $22.8 \pm 6.2 \mathrm{~mm}$ in the group one and to $27.3 \pm 6.2$ in the group two.

In both groups, there was a further regression of pain by the VAS, but, in the period from the $1^{\text {st }}$ to $3^{\text {rd }}$ months from the start of the treatment, in the first group there was a kind of "plateau» or a slowdown. Subsequently, the patients in both groups showed a decrease in pain intensity, after 6 months the VAS values were $11.7 \pm 2.2 \mathrm{~mm}$ in the first and $12.5 \pm 7.6 \mathrm{~mm}$ in the second group. After 12 months, the pain by VAS decreased to $1.1 \pm 2.2 \mathrm{~mm}$ in the first group and $0.7 \pm 0.5 \mathrm{~mm}$ in the second group (Fig. 1). There was no difference in the VAS comparing LR-PRP and LP-PRP treatment in patients from both groups for 12 months.

The mean values of knee functional ability at the beginning of treatment by the Lysholm Knee Score were $72.4 \pm 2.1$ points for patients of the first group and $67.8 \pm 3.2$ points for the second group (Fig. 2). During the first month from the start of the treatment, the Lysholm values in patients after LR-PRP injection increased to $81.6 \pm 4.3$ points, and after LP-PRP increased to $73.3 \pm 3.8$ points. Positive dynamics in both groups maintained throughout the observation period: after 9 months the average value was $93.9 \pm 2.4$ points, after 12 months it was $92.2 \pm 1.7$ points in the first group, as well as $93.9 \pm 2.4$ and $90.2 \pm 2.9$ points after 9 and 12 months, respectively, in the second group of patients.

The mean value of the knee performance at the beginning of the treatment by the Oxford Knee Score was $134.8 \pm 8.8$ for the first group and $138.5 \pm 5.2$ points for the second group. When assessing joint function by the Oxford Knee Score 3 months after the start of the treatment, the mean values were $152.1 \pm 2.3$ points after LR-PRP treatment and $145.7 \pm 5.1$ points after LP-PRP injections.

After 6 months in the first group, the Oxford Knee Score values were $166.0 \pm 5.2$ points with a subsequent increase to $185.5 \pm 2.4$ and $191.2 \pm 5.3$ points after 9 and 12 months. In patients of the second 
group, the values of knee function were $166.0 \pm 6.26,187.0 \pm 4.2$ and $190.0 \pm 6.9$ points after 6,9 and 12 months, respectively.

There was a high negative correlation according to Pearson's correlation coefficient between the values of the VAS and the assessment of the knee functional ability by the Lysholm Knee Score (Fig. 3) and the Oxford Knee Score (Fig. 4) in patients with stage 1 gonarthrosis in both groups. Thus, we can conclude that the decrease in the pain was accompanied by an increase in the knee functional ability.

Fig. 1. The 1-year dynamics of the pain by the VAS in the patients of the groups $1(n=9)$ and $2(n=6)$ with stage 1 gonarthrosis; $M \pm m$.

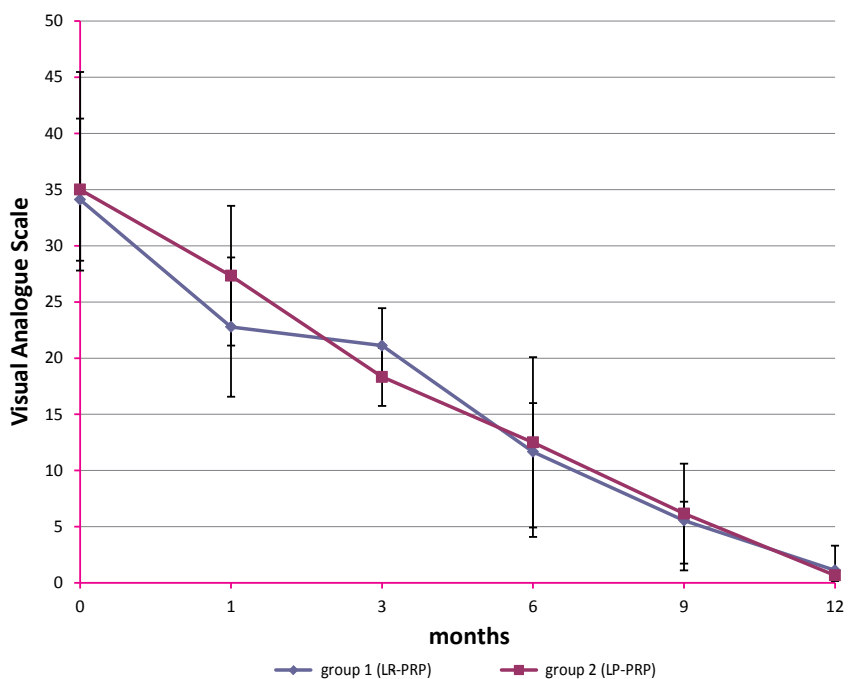

\section{The evaluation of the clinical results in patients with stage 2} gonarthrosis.

In the patients of the first group during a month there was a decrease in pain by the VAS to an average value of $25.5 \pm 9.3 \mathrm{~mm}$ (initial value of $44.5 \pm 11.2 \mathrm{~mm}$ ) and up to $29.4 \pm 9.1 \mathrm{~mm}$ (initial value of $42.5 \pm 12.2 \mathrm{~mm}$ ) in the patients of the second group (Fig. 5).

In both groups, the reduction in pain by the VAS remained for up to 12 months from the start of the treatment, but in the period from 1 to 3 months in the patients of the first group, as at stage 1 gonarthrosis, there was a «plateau», a slowing down of the dynamics for up to three months after the treatment. Subsequently, patients in both groups showed a decrease in pain intensity to $12.0 \pm 2.6 \mathrm{~mm}$ and $12.5 \pm 5.3 \mathrm{~mm}$ in the first and $13.1 \pm 0.5 \mathrm{~mm}$ and $15.0 \pm 0.5$ in the second group after 9 and 12 months, respectively $(p \leq 0.05)$. In the patients from both groups, there was no significant difference in the VAS scores (Fig. 1), comparing the treatment with LR-PRP and LP-PRP for 12 months $(p \geq 0.05)$.

At the beginning of the treatment, the average knee functional ability in patients with stage 2 gonarthrosis by the Lysholm Knee Score for the first and second groups were $64.8 \pm 7.4$ and $65.6 \pm 5.6$ points, respectively. According to the Oxford Knee Score, the values were $116.4 \pm 3.9$ and $110.4 \pm 3.9$, respectively (Fig. 6).

During the first month from the beginning of the treatment, the values by the Lysholm Knee Score in the first group increased to $74.1 \pm 5.1$, and in the second - to $73.3 \pm 4$.3. Subsequently, up to 3 months in the first group there was a slowdown in the dynamics $(75.0 \pm 3.5)$, with a further increase to $84.1 \pm 2.5$ after 6 months and $87.3 \pm 2.5$ after 12 months of the treatment $(p \leq 0.05)$. In the second group, the increase in the knee functional ability was stepless and more steady it was $80.8 \pm 5.1$ after 3 months, $84.4 \pm 3.3$ after 6 months and $87.8 \pm 3.3$ after one year of treatment $(p \leq 0.05)$. In patients from both groups, there was no difference in the improvement of joint function according to the Lysholm Knee Score (Fig. 6 A), comparing treatment with LR-PRP and LP-PRP for 12 months $(p \geq 0.05)$.

Fig. 2. The 1-year dynamics of values of knee function according to the Lysholm Knee Score (A) and the Oxford Knee Score (B) in the studied patients of the group $1(n=9)$ and $2(n=6)$ with stage 1 gonarthrosis; $M \pm m$.

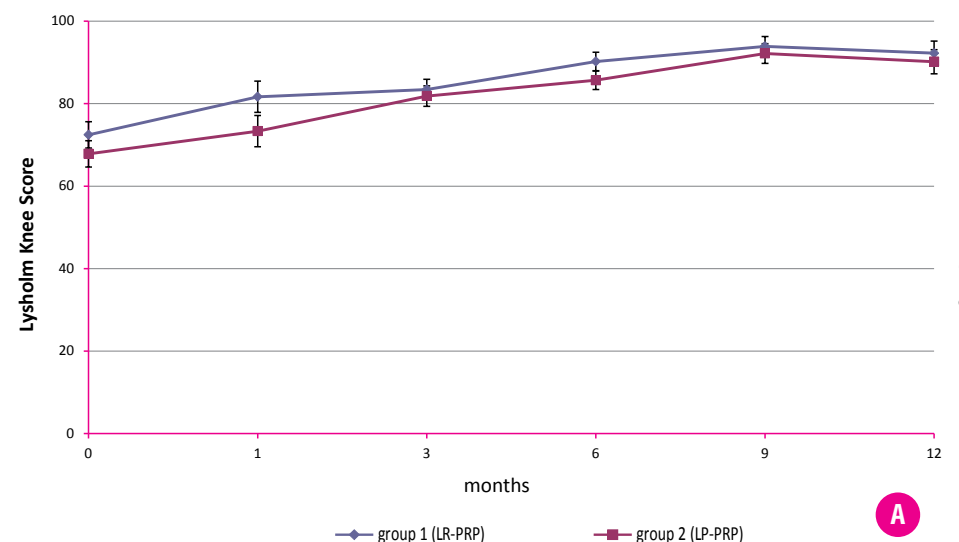

$r=-0.86$

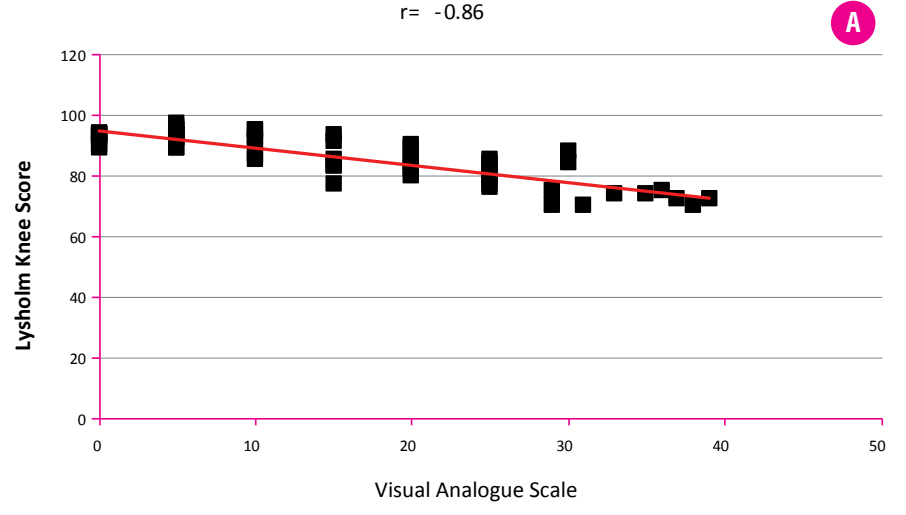

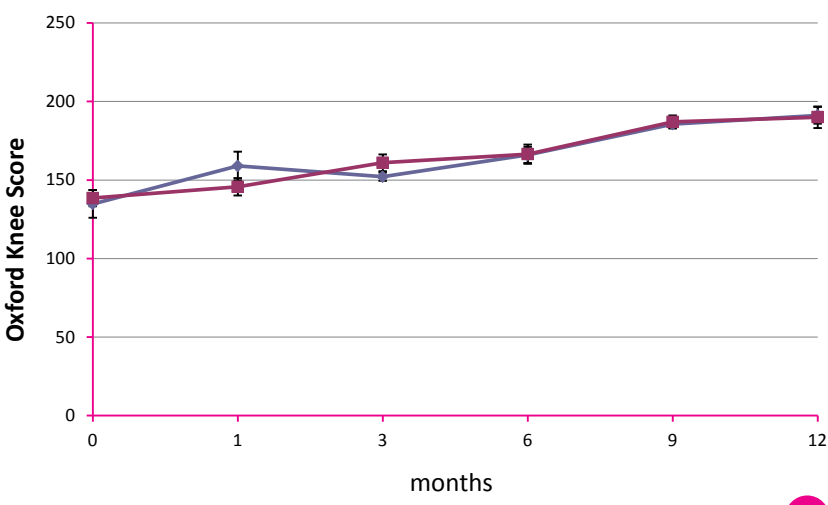

$\rightarrow$ group 1 (LR-PRP) $\quad \rightarrow$ group 2 (LP-PRP)
Fig. 3. Pearson's correlation between the Visual Analogue Scale and the Lysholm Knee Score in the studied patients of group 1 (A) and 2 (B) with stage 1 gonarthrosis.

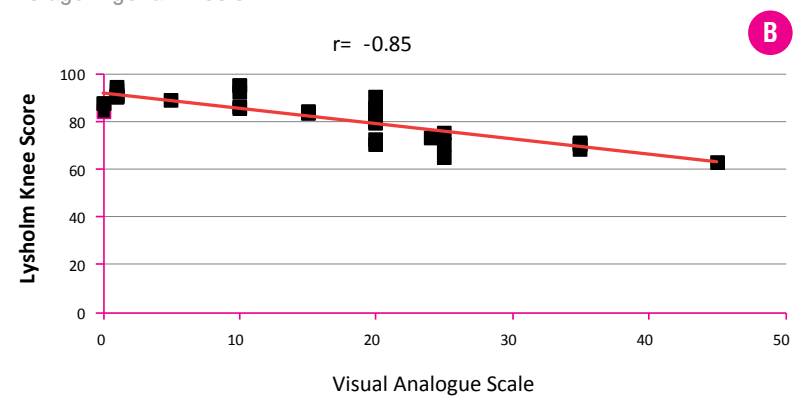


Fig. 4. Pearson's correlation between the Visual Analogue Scale and the Oxford Knee Score in the studied patients of group 1 (A) and 2 (B) with stage 1 gonarthrosis.

$r=-0.87$

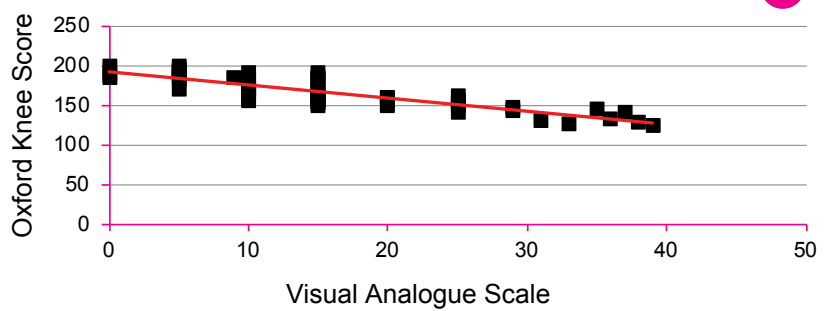

During the first month of the treatment, the values by the Oxford Knee Score in the first group increased to $141.9 \pm 4.8$, in the second they increased to $130.0 \pm 4.1$ (Fig. 6 B). In 3 months, the mean values were 150.5 \pm 8.6 in patients of the first group and $152.8 \pm 4.7$ in the second group. As by the Lysholm Knee Score, in the period up to 3 months there was a slowdown in the dynamics of values in the first group. After 6 months in the first group, the average 0xford Knee Score value was $173.0 \pm 8.6$ with a subsequent increase to $185.6 \pm 4.1$ and $188.4 \pm 7.3$ after 9 and 12 months $(p \leq 0.05)$. In the second group, the increase in knee function according to the Oxford Knee Score was more uniform: $173.0 \pm 3.7$ after 6 months, $182.0 \pm 2.5$ and $185.6 \pm 8.2$ after 9 and 12 months, subsequently $(p \leq 0,05)$. In the patients of the first and second groups, there was no significant difference in the improvement of knee function by the 0xford Knee Score (Fig. 6), comparing the methods of treatment with both types of PRP for 12 months $(p \geq 0.05)$.

There was a high negative correlation according to Pearson's test between pain by the VAS and assessment of the knee functional ability by the Lysholm (Fig. 7) and Oxford (Fig. 8) Knee Scores in the patients with stage 2 gonarthrosis in both study groups. Thus, the increase in the functional knee ability correlated with a decrease in the values of the VAS scale.

\section{The evaluation of the clinical results in patients with stage 3}

\section{gonarthrosis.}

In the patients with stage 3 gonarthrosis, regardless of the cellular product used, a reduction in pain and a slight improvement in knee functional ability were achieved within 6 months. Subsequently, patients in both groups showed a gradual regression of positive symptoms.

In the patients with stage 3 gonarthrosis during the first month there was a decrease in pain by the VAS to an average of $33.8 \pm 6.4 \mathrm{~mm}$ in the first group (55.6 $\pm 11.8 \mathrm{~mm}$ at the beginning) and up to $45.1 \pm 10.0 \mathrm{~mm}$ in the second ( $55.0 \pm 7.1 \mathrm{~mm}$ at the start) (Fig. 9$)(p \geq 0.05)$. In both groups, the positive dynamics of the VAS reduction remained for up to 6 months from the start of the treatment: the values were $26.9 \pm 5.3$ and $25.0 \pm 4.1$ $\mathrm{mm}$, respectively, after which in 12 months there was a gradual increase

A

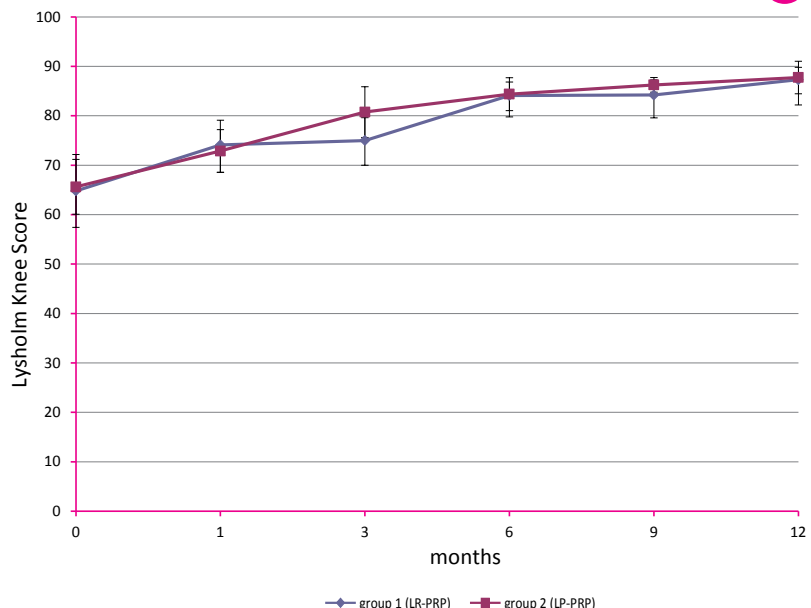

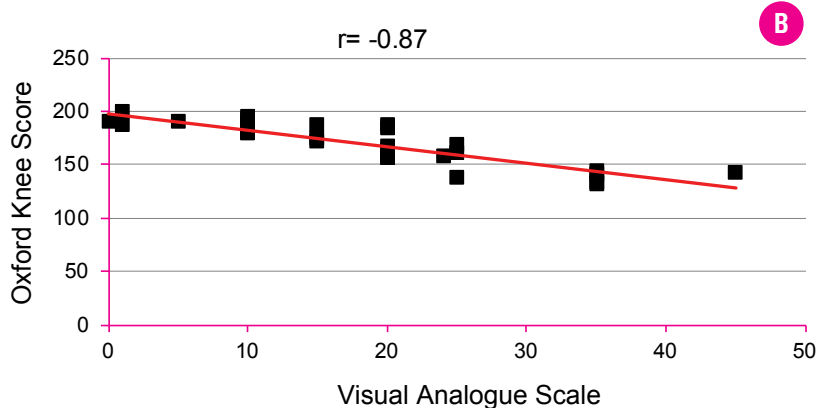

Fig. 5. The 1-year dynamics of the VAS in the group $1(n=10)$ and $2(n=8)$ of patients with stage 2 gonarthrosis; $M \pm m$.

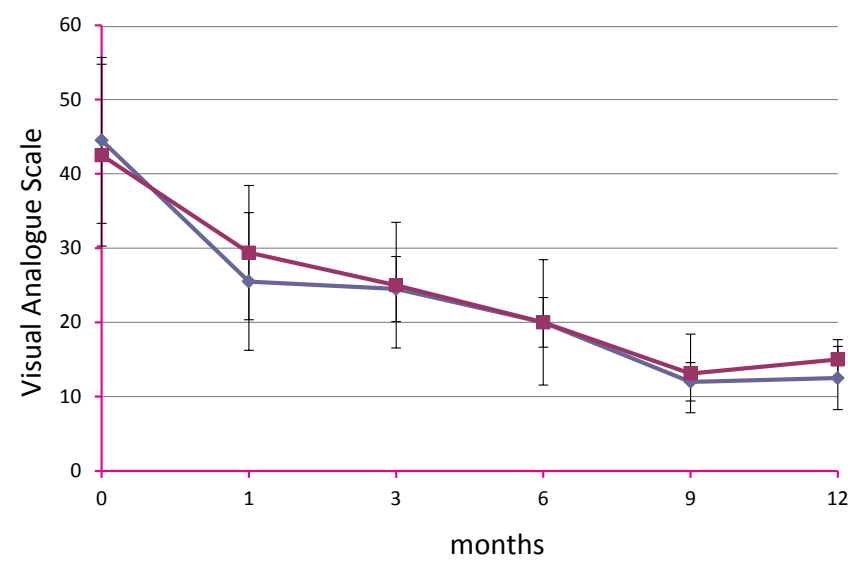

$\multimap \operatorname{group} 1$ (LR-PRP) $\rightarrow$ group 2 (LP-PRP)

of pain to $31.3 \pm 4.4 \mathrm{~mm}$ by the VAS in the first group and $32.1 \pm 2.7 \mathrm{~mm}$ in the second $(p \leq 0.05)$. The patients of both groups had no difference in the reduction in pain by the VAS, comparing LR-PRP and LP-PRP treatment for 12 months $(p \geq 0.05)$.

At the beginning of the treatment, the average knee functional ability in patients with stage 3 gonarthrosis by the Lysholm Knee Score was $52.8 \pm 4.5$ in the first group of patients and $52.4 \pm 4.3$ in the second. According to the Oxford Knee Score, the values were $115.4 \pm$ and $112.3 \pm 7.1$, respectively (Fig. 10)

Evaluation of the knee functional ability in the patients showed positive dynamics with a peak of efficiency within 3 months with an increase to $72.1 \pm 3.3$ points in the first and $70.6 \pm 2.3$ in the second group. It was

Fig. 6. The 1-year dynamics of the values of knee functional ability by the Lysholm Knee Score (A) and the Oxford Knee Score (B) in the patients of groups $1(n=10)$ and $2(n=8)$ with stage 2 gonarthrosis; $M \pm m$.

B

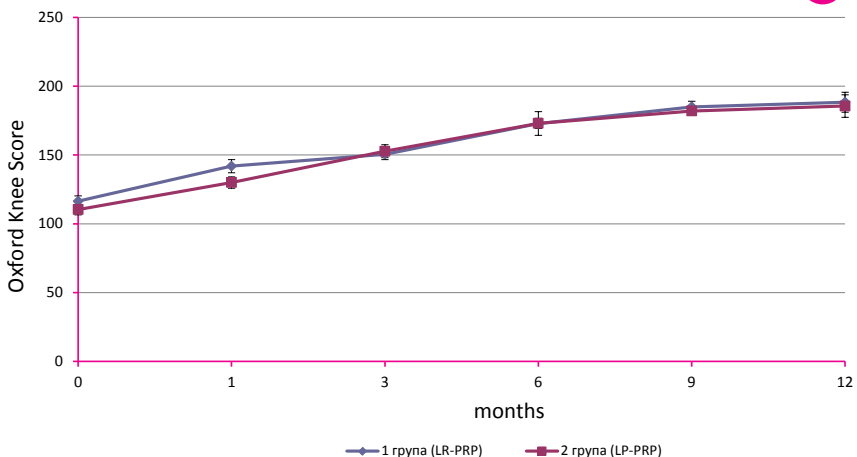


Fig. 7. Pearson's correlation between the Visual Analogue Scale and the Lysholm Knee Score in the studied patients of groups 1 (A) and 2 (B)

with stage 2 gonarthrosis

$$
r=-0.76
$$

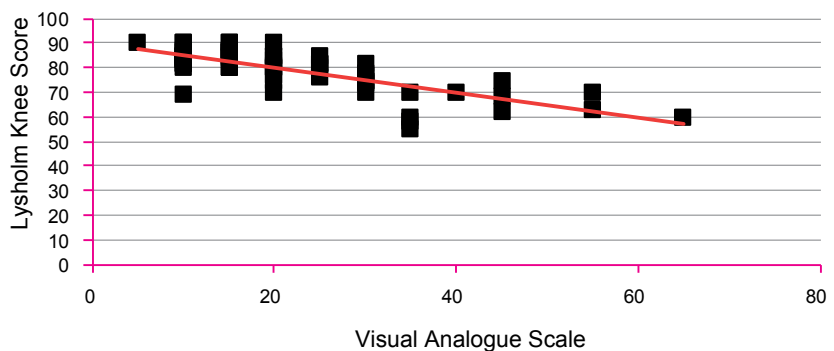

$r=-0.73$

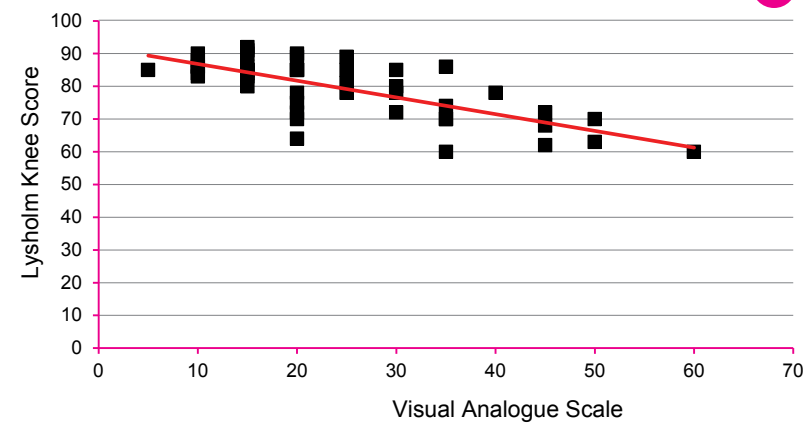

Fig. 8. Pearson's correlation between the Visual Analogue Scale and the Oxford Knee Score in the studied patients of groups 1 (A) and 2 (B) with

stage 2 gonarthrosis. $\quad r=-0.83$

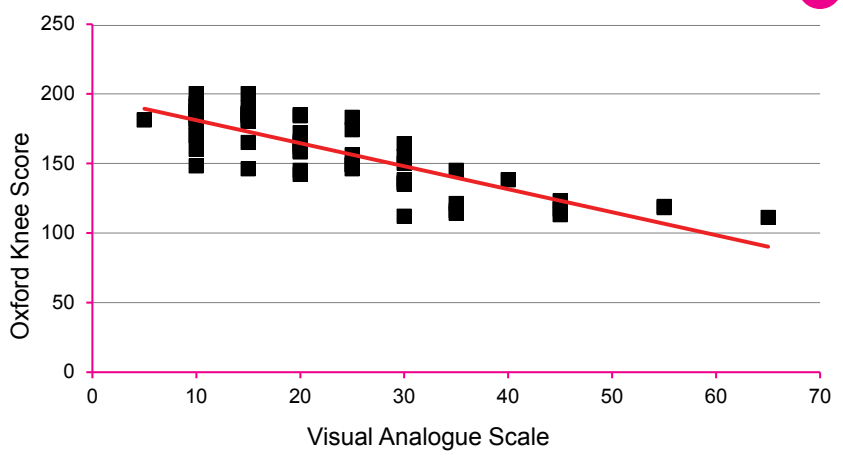

a gradual decrease in the achieved effect to $59.8 \pm 4.9$ and $51.6 \pm 18.7$ points, respectively, in 12 months after the treatment $(p \geq 0.05)$.

According to the Oxford Knee Score, the peak of efficacy was $138.0 \pm 0.7$ points for the first and $123.1 \pm 0.7$ for the second group which also occurred in 3 months from the start of the treatment with subsequent regression. After 12 months, the mean values were $119.8 \pm 8.7$ points in the first and $113.0 \pm 8.7$ in the second group $(p \geq 0.05)$. There was not found a significant difference in the knee functional ability of the joint by the Lysholm and Oxford Knee Scores for 12 months, after comparing the effect of the treatment in patients from both groups $(p \geq 0.05)$.

There was a middle negative correlation according to Pearson's correlation coefficient between pain by the VAS values and assessment of the knee functional ability by the Lysholm (Fig. 11) and Oxford (Fig. 12) Knee Scores in patients with stage 3 gonarthrosis in both study groups. It is possible to make assumptions about the correlation of knee functional ability with decrease in pain by the VAS values.

Comparing the results of the treatment of patients with gonarthrosis of different stages, we can highlight several important points. The main complaint of the patients, regardless of the stage of the disease was pain ( $85 \%$ of patients). Restriction of the movement as an isolated symptom was found only in $12.5 \%$ of cases (5 patients with stage 2 and 1 with stage 3 gonarthrosis). Pain during walking was distinctive for $44 \%$ patients (11 patients with stage 1 gonarthrosis, 10 - with stage 2), constant pain was characteristic of $35 \%$ patients (11 patients with stage 3 gonarthrosis, 4 - with stage 2, 2 - with stage 1). In other cases, the pain was intermittent.

In our opinion, the increased pain during the first days of LR-PRP injection, is directly related to the presence of leukocytes and proinflammatory interleukins in the cellular productwhich enhance inflammation. These cytokines also increase the expression of genes that promote catabolic changes in cartilage tissue and enhance inflammatory processes, including pro-inflammatory interleukins (IL-1 $\beta$, IL-6 and IL-8), inducible NO-synthase (iNOS), cyclooxygenase 2 (COX2) and metalloproteinases 1 and 13 (MMP1, MMP13) [1, 29, 30, 32]. In particular, iNOS is respon-

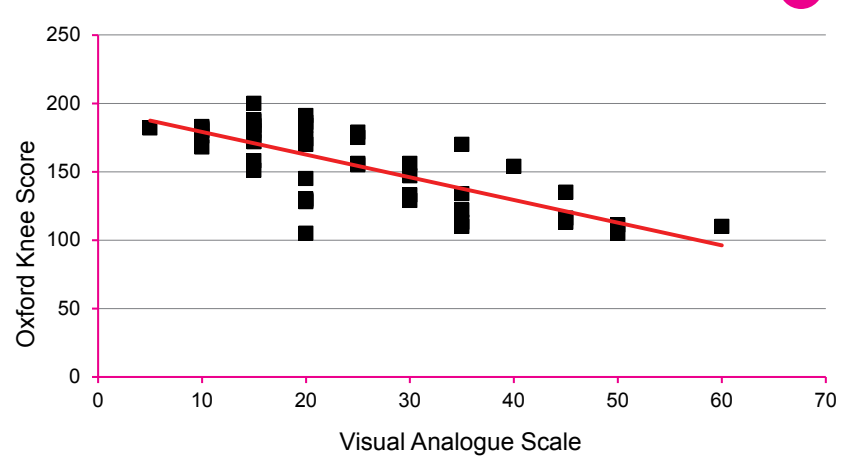

Fig. 9. The 1-year dynamics of the VAS in the studied patients of groups $1(n=8)$ and $2(n=7)$ with stage 3 gonarthrosis; $M \pm m$.

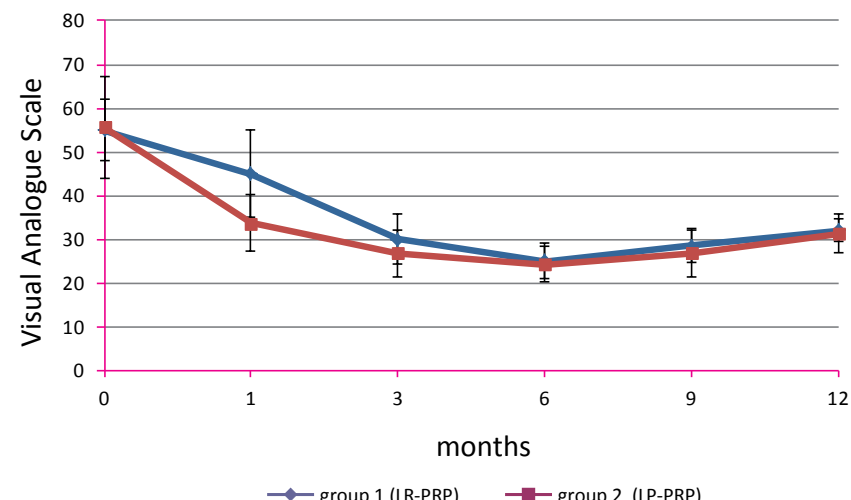

sible for the synthesis of NO that is one of the main pro-inflammatory mediators at osteoarthritis [19]. Increase in the concentration of metalloproteinases MMP1 and MMP13, belonging to collagenases, can cause the destruction of extracellular collagen type II. It is known that increased levels of these metalloproteinases are characteristic of osteoarthritis and rheumatoid arthritis [4].

In addition, proinflammatory cytokines IL-1 $\beta$ and TNF-a reduce the synthesis of the main components of the extracellular matrix in the cartilage tissue of aggrecans and collagen II, increase chondrocyte death by apoptosis and increase the formation of reactive oxygen forms that contribute to oxidative cartilage damage [33]. However, plasma contains a significant amount of anti-inflammatory factors, in particular IL-1 receptor agonist (IL-1Ra). It inhibits the activation of IL-1 by blocking its interaction with its own receptor. In addition, plasma contains a significant amount of anti-inflammatory interleukins IL-4, IL-10 and IL-13. They 
Fig. 10. The 1-year dynamics of the values of knee functional ability by the Lysholm Knee Score (A) and Oxford Knee Score (B) in the studied patients of groups $1(n=8)$ and $2(n=7)$ with stage 3 gonarthrosis; $M \pm m$.

A
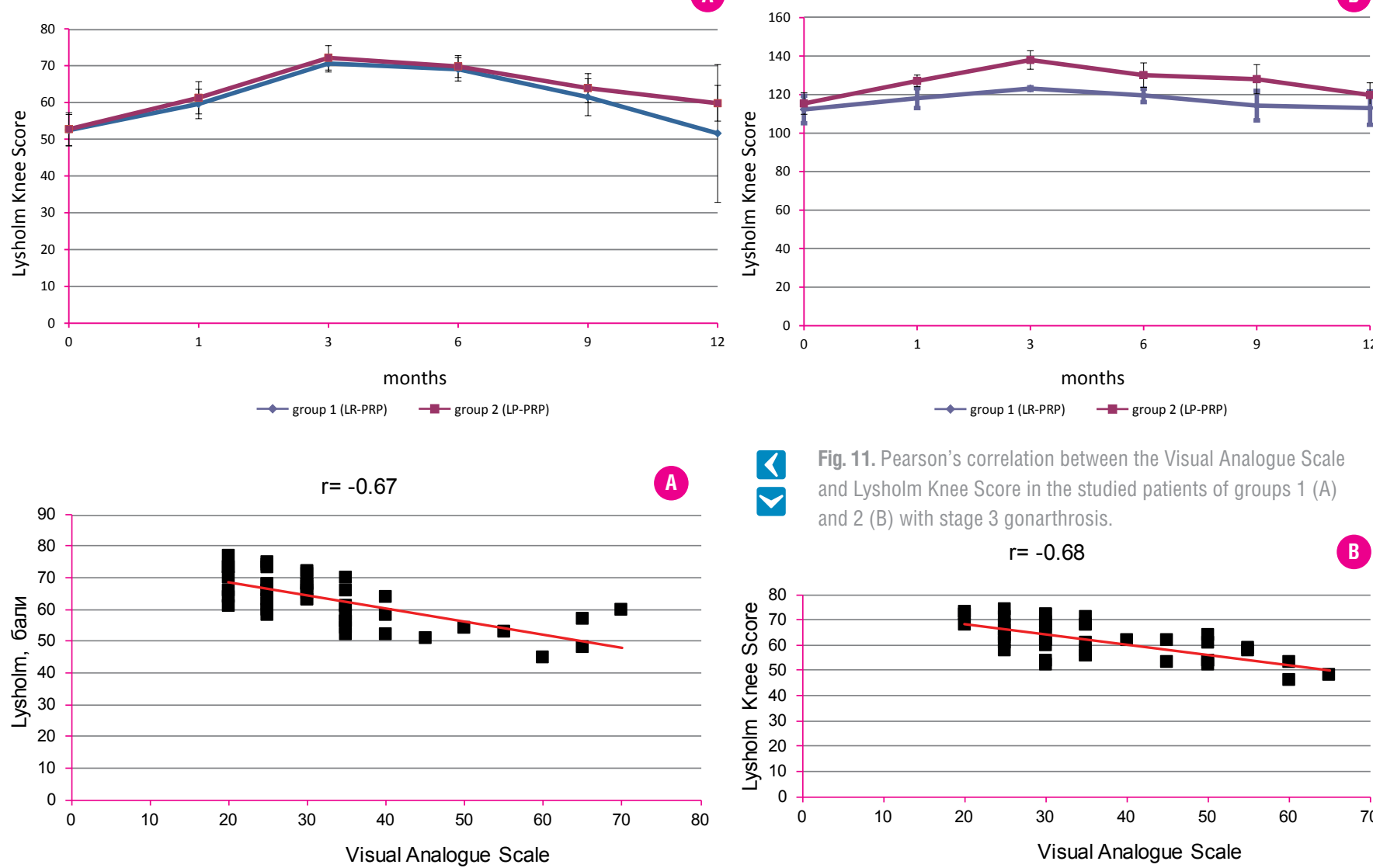

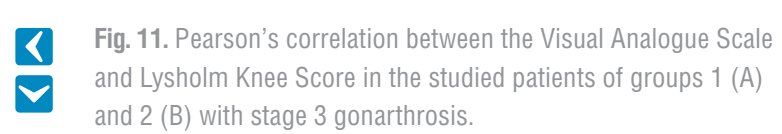
$r=-0.68$

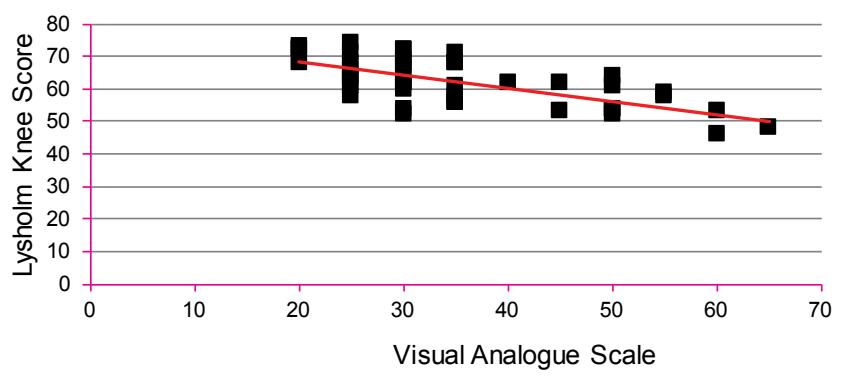

Fig. 12. Pearson's correlation between the Visual Analogue Scale and Oxford Knee Score in the studied patients of groups 1 (A) and 2 (B) with stage 3 gonarthrosis.

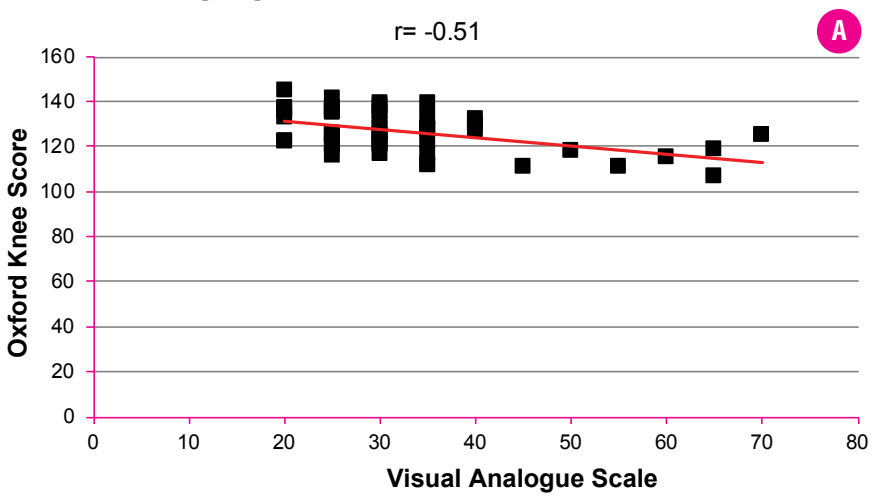

inhibit the synthesis of prostaglandin E2 under the influence of TNF-a. This may explain the gradual and noticeable reduction of pain in patients with different stages of gonarthrosis [17, 28].

Neutrophils are one of the first cells to appear in the area of acute inflammation. This is a response to cytokines such as interleukins (IL-1, IL-8) and TNF-a along with many others. Under physiological circumstances, neutrophils undergo apoptosis and are completely destroyed by tissue macrophages $[2,27]$. Under certain conditions, such as the lack of macrophages, neutrophils undergo necrosis which releases all their intracellular content that causes an increase and prolongation of the inflammatory response.

Monocytes are able to inhibit the presentation of antigens and production of cytokines. Usually, LR-PRP contains a significantly increased

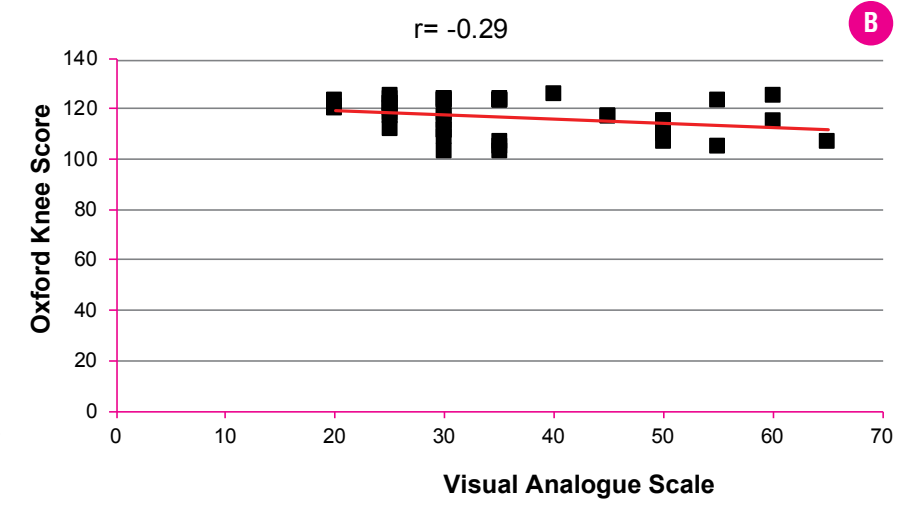

(up to 3-6 times from baseline) concentration of monocytes, and an increased concentration of platelets can stimulate the migration of mononuclear cells [11,31]. This should neutralize the effects of significant concentrations of neutrophils but some studies suggest that it also causes an increase in the concentration of proinflammatory interleukins which enhance the inflammatory response in the early period. In addition, monocytes migrate to the site of the lesion and differentiate into macrophages and dendritic cellswhich initiates, in contrast to neutrophils, a long-lasting immune response (months, but not days compared to neutrophils) [18, 32]. However, the frequency and severity of pain in the first few days after injection were significantly higher than in patients after LP-PRP regardless of the stage of the disease in the group of patients that received LR-PRP treatment $(p \leq 0.05)$. In the same group of patients, the clinical 
effect appeared in an earlier period (after 1-2 injections compared with 4-5 injections) than after the use of LP-PRP ( $p \leq 0.05)$.

After using LR-PRP treatment in patients with stage 1 and 2 gonarthrosis, there was a typical decrease in the achieved clinical effect within 1-3 months and reaching a «plateau». Patients noted a slowdown in the reduction of clinical manifestations such as pain, joint stiffness, edema, synovitis, etc. However, these phenomena were temporary and the exa-mination 6 months after the start of treatment revealed positive dynamics in comparison with both the baseline and previous periods of observation $(p \leq 0.05)$. In our opinion, the reduction of pain led to a shortterm improvement in the knee functional ability in the patients with stage 3 gonarthrosis.

The slowdown in the dynamics of 1-3 months is typical for patients receiving LR-PRP. In our opinion, it is associated with the activation of another pathway for the implementation of the anti-inflammatory effect, namely activation of endogenous multipotent mesenchymal stromal cells (MMSCs). Comparing with LR-PRP treatment, a positive therapeutic effect was observed in the patients a bit later (3-4 weeks) after the start of treatment. The activation of MMSCs is partially mediated by the chemokine stromal cell-derived factor-1a (SDF-1a, also known as CXCL12) contained in platelet alpha granules. This effect of enhancing the migration of progenitor cells is used in the treatment of osteoarthritis and cartilage defects such as microfracture, tunneling during surgery. The lack of pronounced and long-term clinical effect in patients with stage 3 gonarthrosis, compared with patients with stage 1-2, can be explained by de- generative changes in joint and periarticular tissues, severe subchondral sclerosis, trophic disorders that limit the clinical effectiveness of the PRP therapy and prevent proliferation and migration of MMSCs. At the same time, the stimulation of regenerative processes, even in such an unfavorable situation, allowed to achieve a significant reduction in pain, the severity of clinical symptoms and improved the quality of patients' life.

In addition, abundant platelet vascular endothelial growth factor (VEGF) and platelet-derived growth factor (PDGF) promote the migration of MMSCs as well as macrophages and fibroblasts [3, 8, 21].

Yin W. et al. concluded that at the same platelet concentration in LPPRP and LR-PRP, a higher concentration of leukocytes causes the activation of different signaling pathways of regeneration [29]. In the model of osteoarthritis in laboratory animals, LR-PRP caused a significant increase in the level of proinflammatory cytokineswhich negatively affected the regeneration processes, cartilage metabolism and counteracted the positive effects of growth factors [29].

Other studies have also demonstrated the benefits of using LP-PRP in the treatment of knee osteoarthritis at various stages and its ability to recruit MMSCs into regeneration processes $[7,9,10,20]$. The opposite data are demonstrated in the study of Oltulu I. et al., the best results were obtained in the group of patients after the use of LR-PRP [23]. However, in this study, the cell composition draws attention, namely a significantly higher concentration of platelets in LR-PRP compared to LP-PRP and almost unchanged concentration of leukocytes in LP-PRP compared to baseline.

\section{CONCLUSION}

The assessment of treatment outcomes after 12 months compared to baseline in stages 1 and 2 gonarthrosis showed a positive result for both groups of patients after using LR-PRP and LP-PRP treatment, and in stage 3 a significant improvement was achieved for the period up to 6 months. There was no significant difference between the results of LR-PRP and LP-PRP treatments in the periods of 9-12 months.

After using LP-PRP injections, the 1-year dynamics of quality of life was stepless and more steady without periods of regression while for LRPRP treatment there was a clear significant short-term reduction in the effect of treatment for about 6 months compared to both the baseline and values of the previous period in the $1^{\text {st }}$ and $2^{\text {nd }}$ stage of the disease with further improvement and stable effect for up to 12 months.

\section{REFERENCES}

1. Assirelli E, Filardo G. Effect of two different preparations of platelet-rich plasma on synoviocytes. Knee Surg. Sports. Traumatol. Arthrosc. 2015; 23(6):2690-2703. DOI: 10.1007/s00167-014-3113-3

2. Borregaard N. Neutrophils, from marrow to microbes. Immunity. 2010; 33(5):657-70. DOI: 10.1016/j.immuni.2010.11.011. PMID: 21094463.

3. Caplan Al, Correa D. PDGF in bone formation and regeneration: New Insights into a Novel Mechanism Involving MSCs. J Orthop Res. 2011. 2011:1795-1803. D0I: 10.1002/jor.21462.

4. Chubinskaya $S$, Kuettner KE, Cole $A A$. Expression of matrix metalloproteinases in normal and damaged articular cartilage from human knee and ankle joints. Lab Invest. 1999; 79(12):1669-77. PMID: 10616215.

5. DeLong JM, Russell RP, Mazzocca AD. Platelet-rich plasma: the PAW classification system. Arthroscopy. 2012; 28(7):998-1009. D0I:10.1016/j.arthro.2012.04.148.

6. Dohan Ehrenfest DM, Rasmusson L, Albrektsson T. Classification of platelet concentrates: from pure platelet-rich plasma (P-PRP) to leucocyte- and platelet-rich fibrin (L-PRF). Trends Biotechnol. 2009; 27(3):158-67. DOl:10.1016/j.tibtech.2008.11.009.

7. Duif C, Vogel T, Topcuoglu F, Spyrou G, von Schulze Pellengahr C, Lahner M. Does intraoperative application of leukocyte-poor platelet-rich plasma during arthroscopy for knee degeneration affect postoperative pain, function and quality of life? A 12-month randomized controlled double-blind trial. Arch Orthop Trauma Surg. 2015; 135(7):971-7. D0I:10.1007/s00402-015-2227-5.

8. Eppley BL, Woodell JE, Higgins J. Platelet quantification and growth factor analysis from platelet-rich plasma: implications for wound healing. Plast Reconstr Surg. 2004; 114(6):1502-1508. DOI:10.1097/01.prs.0000138251.07040.51.

9. Filardo G, Kon E, Di Marino A, Sessa A, Merli M, Marcacci M, et al. Leukocyte-poor PRP application for the treatment of knee osteoarthritis. Joints. 2013; 01(03):112-20.

10. Filardo G, Previtali D, Napoli F. Candrian C, Zaffagnini S, Grassi A. PRP injections for the treatment of knee osteoarthritis: A meta-analysis of randomized controlled trials. Cartilage. 2020. DOI: $10.1177 / 1947603520931170$.

11. Fitzpatrick J, Bulsara MK, McCrory PR, Richardson MD, Zheng MH. Analysis of Platelet-Rich Plasma Extraction: Variations in Platelet and Blood Components Between 4 Common Commercial Kits. Orthopaedic journal of sports medicine. 2017; 5(1):1-8. DOl.org/10.1177/2325967116675272.

12. Furman MI, Barnard MR, Krueger LA, Fox ML, Shilale EA, Lessard DM. Circulating Monocyte-platelet aggregates are an early marker of acute myocardial infarction. J Am Coll. Cardiol. 2001; 38:1002-6. DOI: 10.1016/s0735-1097(01)014851. 
13. Htun P, Fateh-Moghadam S, Tomandl B, Klinger K, Stellos K, Garlichs $C$. Course of platelet activation and platelet-leukocyte interaction in cerebrovascular ischemia.Stroke. 2006; 37:2283-7. DOI:10.1161/01.STR.0000236638.75591.61.

14. Joseph JE, Harrison P, Mackie IJ, Isenberg DA. Increased circulating plateletleucocyte complexes and platelet activation in patients with antiphospholipid syndrome, systemic lupus erythematosus and rheumatoid arthritis. Brithsh J. Haematol. 2001; 115:451-9. D0l: 10.1046/j.1365-2141.2001.03101.

15. Kikuchi N, Yoshioka T, Taniguchi $Y$, Sugaya $H$, Arai N, Kanamori A, et al. Optimization of leukocyte-poor platelet-rich plasma preparation: a validation study of leukocyte-poor platelet-rich plasma obtained using different preparer, storage, and activation methods. J Exp Orthop. 2019; 6(1):24. D0I:10.1186/s40634-0190190-8.

16. Kobayashi $Y$, Saita $Y$, Nishio H, lkeda H, Takazawa Y, Nagao M, et al. Leukocyte concentration and composition in platelet-rich plasma (PRP) influences the growth factor and protease concentrations. J Orthop Sci. 2016; 21(5):683-9. DOI:10.1016/j.jos.2016.07.009.

17. Kennedy MI, Whitney K, Evans T, LaPrade RF. Platelet-rich plasma and cartilage repair. Curr Rev Musculoskelet Med. 2018; 11(4):573-82. D0I:10.1007/s12178018-9516.

18. Lana JF, Huber SC, Purita J, Tambeli CH, Santos GS, Paulus C, et al. Leukocyte-rich PRP versus leukocyte-poor PRP - The role of monocyte/macrophage function in the healing cascade. J Clin Orthop Trauma. 2019; 10(Suppl 1):S7-12. DOI:10.1016/j.jcot.2019.05.008.

19. Leonidou A, Lepetsos $P$, Mintzas $M$, Kenanidis E, Macheras $G$, Tzetis $M$, et al. Inducible nitric oxide synthase as a target for osteoarthritis treatment. Expert Opin Ther Targets. 2018; 22(4):299-318. DOI:10.1080/14728222.2018.1448062.

20. Lucarelli E, Beccheroni A, Donati D, Sangiorgi L, Cenacchi A, Del Vento AM, et al. Platelet-derived growth factors enhance proliferation of human stromal stem cells. Biomaterials. 2003; 24(18):3095-100. D0I:10.1016/S0142-9612(03)00114-5.

21. Meeson R, Sanghani-Keri A, Coathup M, Blunn G. VEGF with AMD3100 endogenously mobilizes mesenchymal stem cells and improves fracture healing. J Orthop Res. 2019; 37(6):1294-302. DOI: 10.1002/jor.24164.

22. Mickelson JK, Lakkis NM, Villarreal-Levy G, Hughes BJ, Smith CW. Leukocyte activation with platelet adhesion after coronary angioplasty: a mechanism for recurrent disease? J Am Coll Cardiol. 1996; 28(2):345-53. DOl:10.1016/0735-1097(96)00164-7.

23. Oltulu, I, Korkmaz O, Isyar M, Kara A, Bulbul AM., Mahirogulları M. Does the Difference in Leukocyte Concentration of PRP Affect the Short-Term Follow-Up Results in Cases Diagnosed with Early Stage Knee Osteoarthritis? Serbian J Exp Clin Res. 2019. DOI:10.2478/sjecr-2019-0010.

24. Ott I, Neumann FJ, Gawaz M, Schmitt M, Schömig A. Increased neutrophil-platelet adhesion in patients with unstable angina. Circulation. 1996; 94(6):1239-46. DOI:10.1161/01.cir.94.6.1239

25. Pifer MA, Maerz T, Baker KC, Anderson K. Matrix metalloproteinase content and activity in low-platelet, low-leukocyte and high-platelet, high-leukocyte platelet rich plasma (PRP) and the biologic response to PRP by human ligament fibroblasts. Am J Sports Med. 2014; 42(5):1211-8. D0l:10.1177/0363546514524710.

26. Singh MV, Davidson DC, Kiebala M, Maggirwar SB. Detection of circulating platelet-monocyte complexes in persons infected with human immunodeficiency virus type-1. J Virol Methods. 2012; 181(2):170-6. DOl:10.1016/j.jviromet.2012.02.005.

27. Stark MA, Huo Y, Burcin TL, Morris MA, Olson TS, Ley K. Phagocytosis of apoptotic neutrophils regulates granulopoiesis via IL-23 and IL-17. Immunity. 2005; 22(3):285-94 DOI:10.1016/j.immuni.2005.01.011.

28. Xie X, Zhang C, Tuan RS. Biology of platelet-rich plasma and its clinical application in cartilage repair. Arthritis Res Ther. 2014; 16(1):204. DOl: 10.1186/ar4493.

29. Yin W-J, Xu H-T, Sheng J-G, An Z-Q, Guo S-C, Xie X-T, et al. Advantages of pure platelet-rich plasma Compared with leukocyte- and platelet-rich plasma in treating rabbit knee osteoarthritis. Med Sci Monit. 2016; 22:1280-90. D0I:10.12659/msm.898218.

30. Yin W, Qi X, Zhang Y, Sheng J, Xu Z, Tao S, et al. Advantages of pure platelet-rich plasma compared with leukocyte- and platelet-rich plasma in promoting repair of bone defects. J Transl Med. 2016; 14(1):73. D0I:10.1186/s12967-016-0825-9.

31. Yoshida R, Murray MM. Peripheral blood mononuclear cells enhance the anabolic effects of platelet-rich plasma on anterior cruciate ligament fibroblasts: PBMCs and prp stimulate fibroblasts. J Orthop Res. 2013; 31(1):29-34. D0l:10.1002/jor.22183.

32. Wang $S-Z$, Fan $W-M$, Jia J, Ma $L-Y, Y u J-B$, Wang $C$. Is exclusion of leukocytes from platelet-rich plasma (PRP) a better choice for early intervertebral disc regeneration? Stem Cell Res Ther [Internet]. 2018; 9(1). DOl:10.1186/s13287-018-0937-7.

33. Wojdasiewicz $P$, Poniatowski $Ł A$, Szukiewicz $D$. The role of inflammatory and anti-inflammatory cytokines in the pathogenesis of osteoarthritis. Mediat Inflamm. 2014; 2014. DOI: $10.1155 / 2014 / 561459$.

34. Wong VW, Wan DC, Gurtner GC, Longaker MT. Regenerative surgery: tissue engineering in general surgical practice. World J Surg. 2012; 36(10):2288-99. DOl:10.1007/s00268-012-1710-1.

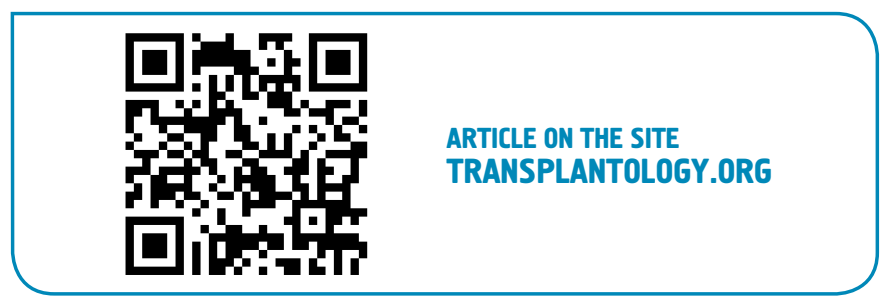

The authors declared no potential conflicts of interest with respect to the research, authorship, and/or publication of this article. 